\title{
An Improved CPE Localization Algorithm for Wireless Sensor Networks
}

\author{
Jianmin Zhang, Hua Li and Jian Li \\ College of Computer, Henan Institute of Engineering \\ Zhengzhou 451191, China \\ zjm1996@163.com,xzhn_2008@163.com,lj2006@163.com
}

\begin{abstract}
Localization is increasingly becoming the focus of research in the filed of Wireless Sensor Networks (WSNs), as the data reported from a sensor is only useful when the position of that sensor is found. In this paper, we proposed an improved CPE (Convex Position Estimation) localization algorithm that reduces the localization error without requiring additional hardware and computational costs. In the proposed scheme, we first estimate the location of sensor nodes using Convex Position Estimation (CPE), and then refine the location of sensor nodes using the location of the two-hop anchor nodes. The simulation results show that the new localization algorithm effectively improves the positioning accuracy compared with the traditional CEP localization algorithms.
\end{abstract}

Keywords: wireless sensor networks, localization, convex position estimation

\section{Introduction}

Recent technological advances have enabled the development of low-cost, lowpower, and multifunctional sensor nodes. These sensor nodes build the wireless sensor networks (WSNs) and perceive the information which in available from the surrounding environment [1]. WSN can be used in various fields, such as military affairs, target tracking, environmental monitoring, disaster management, intelligent transportation, smart home applications, medical care, reconnaissance, and so forth [2]. WNS intends to provide information on spatiotemporal characteristics of the observed physical world. Hence, it is necessary to associate sensed data with locations, making data geographically meaningful. Finding the location of the sensor nodes where the event occurred is an intrinsic and integral part of any WSN application because without finding the position of the sensor that is reporting the sensor that is reporting the sensed data, the latter will not be useful. Besides, location information also supports fundamental network layer services, such as topology control, routing, clustering, and so on [3]. Hence, Localization, a mechanism for autonomously discovering and establishing spatial relationships among sensor nodes, is of great importance in the development of wireless sensor networks.

An approach to find the location of the sensor nodes is to equip it with a built-in GPS receiver [4]. However, this method is totally not suitable for WSNs because sensor nodes are intended to be low-cost disposable devices, and GPS receivers are expensive and power-consuming which are inadequate for the hardware and power-limited sensors. Other approaches, referred to as collaborative localization algorithms, assume that only a small fraction of sensor have their position through manual configuration or using GSP receiver [5]. These sensor nodes are called anchors, and their position can be used as references to estimate the position of other sensors. 
Now, many studies have been conducted on the localization problems in WSNs [8-17]. On the basis of the used information, these localization algorithms can be divided into two categories: range-based algorithms and range free-based algorithms [6, 7]. Range-based approaches are absolute point-to-point distance or orientation between neighbor nodes to estimate the localization of unknown nodes. The measuring technologies in the Rangebased category consists of time difference of arrive (TDOA) [8], time of arrival (TOA) [9], received signal strength indicator (RSSI) [10], and angle of arrival (AOA) [11]. The range-based localization algorithms yield relative precise, but these algorithms need use additional hardware, thus making them more expensive. On the other hand, range-free based algorithms do not require distance or angle information. Thus, the range-free methods do not require any additional costly hardware, which makes it more effective and simpler alternative over range-based approaches. Although range-free schemes provide less accurate results compared with range-based approaches, which is due to ranging errors, they can still satisfy many applications requirements. Because of its costeffectiveness, simplicity, and acceptability in applications, range-free approaches have received greater attention for localization in WSNs, such as Centroid localization algorithm [12], CPE localization algorithm [13], DV-Hop localization algorithm [14], APIT localization algorithm [16], MDS-MAP localization algorithm [17], and so on.

Among many of range-free localization algorithms, CPE algorithm [13] is a neat scheme which worth further investigation. The advantages of the CPE algorithms are its simplicity and the fact that it does not depend on measurement error. However, one of the drawbacks of the CPE algorithm is that the accuracy of the location estimation is somewhat poor. To address this problem, in this paper, we present an improved CPE localization algorithm. The proposed scheme can achieve higher localization accuracy under the same conditions without employing any extra hardware.

The rest of the paper is organized as follows: Section II presents the CPE algorithm and its error analysis. In section III, the improved CPE algorithm is described in detail. In Section IV, simulation results are shown and the performance results are compared with the CPE algorithms. Finally, Section V draws a conclusion.

\section{Overview of the CPE Algorithm}

The convex position estimation (CPE) was proposed by proposed by Doherty et al. The basic idea of the CPE is that if a sensor node can communicate with another sensor, its position is restricted by the connectivity constraints to be in some region relative to the other sensors. Many such connectivity or proximity constraints define the set of feasible sensor position in a WSN. These constraints can be represented as Linear Matrix Inequalities (LMI-s). Once all the constraints in the network are expressed in this form, the LMI-s can be combined to form a single semi definite program. This is soled to produce a bounding region for each node, which Doherty et al., simplify to be a bounding box. If an unknown node can communicate with some neighbouring anchor nodes, then there are connectivity constrains between the unknown node and its neighbouring anchor nodes. Since the location of the unknown node must within the overlapping region of the communication regions of these anchor nodes, the information such as locations and communication ranges of these nearby anchor nodes can be employed to estimate the location of the unknown node.

In order to reduce the calculation the $\mathrm{CPE}$ algorithm define the estimative rectangle (ER) which bounds the overlapping region and regards the centre of the rectangle as the estimative location of the unknown node. The four side of the ER are parallel to the $x$-axis and the $y$-axis. For example, in Figure 1, the unknown node $N$ is with the communication range $r$ of three neighbouring anchor nodes $A_{1}, A_{2}$ and $A_{3}$. That is, there are connectivity constrains between the unknown node $N$ and its 
neighbouring anchor nodes $A_{1}, A_{2}$ and $A_{3}$. Then, by utilizing combinations of this connectivity constrains, the ER of the overlapping region where a unknown node $N$ resides can be calculated.

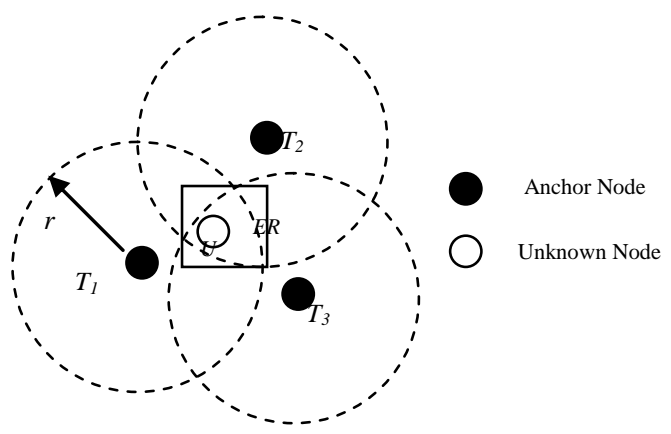

Figure 1. The CPE Algorithm

The CPE algorithm is centralized localization scheme since each unknown sensor node sends the collected connectivity constraints back to a centralized controller. The centralized controller then estimates the location of every unknown node and flood the estimative location back to every unknown node. This central method makes the traffic-load in CPE heavy and the CPE algorithm scale poorly.

In comparison with other localization algorithms, the most attractive feature of the CPE is its simplicity. However, the mean localization error in the CPE algorithm is a monotonically decreasing function of the ratio of anchor nodes to all nodes in the WSN. When the ratio of anchor nodes is small, the estimated location is as poor as a random guess of the node's coordinates. In the following section, we propose a localization algorithm using two-hop anchor nodes algorithms to reduce the estimate error of the nodes when the percentage of anchor nodes is small.

\section{The Improved CPE Algorithm.}

In this section, we present the improved CPE localization algorithm in details. There are three main steps in the improved CEP localization algorithm: Getting the information of the one-hop and two two-hop away neighboring anchor nodes of unknown nodes, getting the initial estimative location of unknown nodes and refining the initial location of unknown nodes.

Algorithm 1: Getting the information of the one-hop and two two-hop away neighboring anchors

1: Each unknown sensor constructs two empty lists List 1 and List 2

$I^{*}$ this two lists are used to keep the ID, position, hop of its one-hop and two hop anchors respectively. hop=0. */

2: Each anchor broadcasts MSG(ID, position, hop) /* hop $=0 /$

3: A timer starts

4: while the timer is not over do

5: for each MSG received by an unknown node receive.

6: if the ID not in the List 1 or List 2 of the unknown node then

I* ID is that of in the received MSG*/

7: $\quad$ if hop=0 then $/ *$ hop is that of in the received MSG)

8: $\quad$ Add the anchor to the List 1

9: $\quad$ Broadcast MSG(ID, position, hop=1)

/* ID and position are that of in the received MSG/ 


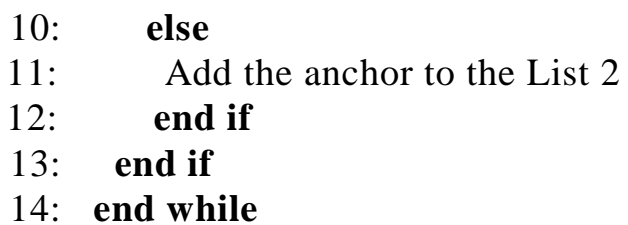

\subsection{Getting the Information of the One-hop and Two Two-hop Away Neighboring Anchors of Unknown Nodes}

In the anchor exchange phase, every sensor node gathers the location information of anchor nodes, which is one-hop and two-hop away via anchor nodes two-hop flooding. By using two-hop flooding, every unknown node can gather the ID and the location information of its one-hop and two-hop neighboring anchor nodes. The process is shown in Algorithm 1.

\subsection{Getting the Initial Estimative Location of Unknown Nodes}

After finishing the anchor exchange phase, all the unknown nodes get the ID and the position of their one-hop and two-hop away anchor nodes. Then each unknown node computes its estimative rectangle (ER) as in CPE algorithm, and then uses the centre of the estimative rectangle as the estimative location of the unknown nodes. This process is the same as that of the CPE localization algorithm, so we don't discuss it here.

\subsection{Refining the Location of Unknown Nodes}

In this phase, the initial estimative location obtained by utilizing estimative rectangle can be further refined by the information of neighbouring two-hop away anchor nodes.

We know that a unknown that a unknown node can't be in the communication range of the it's two-hop anchor node. From Figure 2 we can clearly see that the area of the unknown node be possible in will be reduced by using the information of the two-hop anchor nodes. Figure 2 gives the impact of the two-hop away anchor nodes. When the communication ranges of the two-hop away anchor nodes overlap more with the unknown node's overlapping region, the more impact made by the two-hop away anchor node on the initial estimative location of the unknown node.

In Figure 2, $U$ is an unknown node. $T_{1}$ and $T_{2}$ are two anchor nodes which are two hop away from the unknown node $U$. $U^{\prime}$ is the position of unknown node $U$ computed by using CPE methods. The Line $L_{l}$ is the straight line which through the anchor node $T_{l}$ and the initial estimative location $U^{\prime} \cdot A_{l} B_{1}$ is the segment of line $L_{l}$, which is within the intersection are between the one-hop away anchor nodes' overlapping region and the communication range of the two-hop away anchor node $T_{1}$. And the Line $L_{2}$ is the straight line which through the anchor node $T_{2}$ and the initial estimative location $U^{\prime} . A_{2} B_{2}$ is the segment of line $L_{2}$, which is within the intersection are between the one-hop away anchor nodes' overlapping region and the communication range of the two-hop away anchor node $T_{2}$.

The position the unknown node $N$ can be calculated as:

$$
P=P^{\prime}+\sum_{i=1}^{m} \overrightarrow{A_{i} B_{i}}
$$

where $P^{\prime}$ the initial estimative location of unknown node $N$, which is computed by using CPE method in the former subsection. 
$\overrightarrow{A_{i} B_{i}}$ is a vector, of which the direction of is from two-hop away anchor node to the initial estimative location $N$ '.

$m$ is the number of two-hop away anchor nodes of the unknown node $N$.

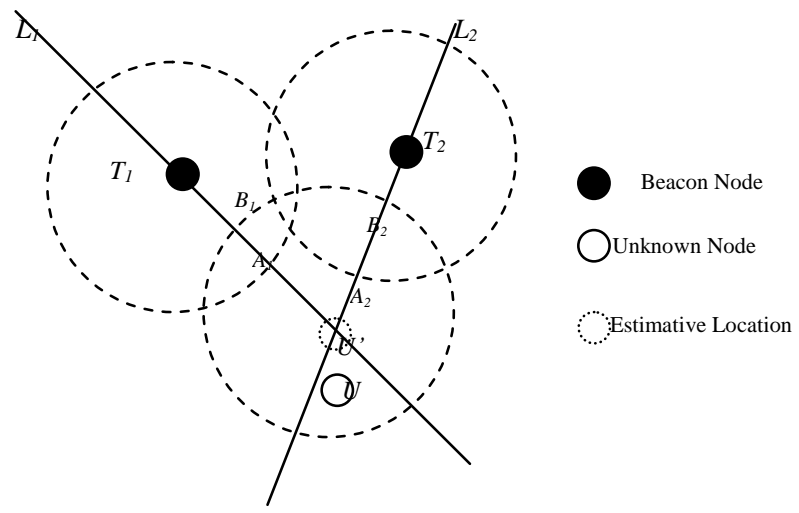

Figure 2. The Proposed Scheme Algorithm

The Formula (1) shows that the larger the communication ranges of the two-hop away anchor nodes overlap with the one-hop away anchor nodes' overlapping region the more affect of the two-hop away anchor nodes on the position of the unknown nodes.

\section{Simulation Result and Analysis}

In this Section, a simulation is done by comparing the improved CPE algorithm presented in this paper with CPE localization algorithm. Simulations are conducted on MATLAB. In the simulations, we use the localization error formula to evaluate the overall performance of the algorithm. The Equation is as follows:

In this simulation, the localization error is defined as the average error function illustrated as follows equalization.

$$
\text { error }=\frac{\sum_{i=M+1}^{N} \sqrt{\left(x_{i}-X\right)^{2}+\left(y_{i}-Y_{i}\right)^{2}}}{R \times(N-M)}
$$

Among them, $N$ is the number of sensor nodes, $\mathrm{M}$ is the number of beacon nodes, $\left(X_{i}, \mathrm{Y}_{i}\right)$ is the actual coordinate of the unknown node $i$ is, $\left(x_{i}, y_{i}\right)$ is the evaluated coordinate, and $R$ is the communication range of sensor nodes. The localization error reflects the accuracy of localization algorithm. The less the localization error is, the more accurate the localization performance.

To attain a better statistics result, the simulations of algorithms are randomly run 200 times for each result. Relevant simulation parameters are outlined below.

- The network region is assumed to be a two dimensional area of $200 \mathrm{~m} \times 200 \mathrm{~m}$. The sensor nodes are distributed randomly in this region.

- Each sensor node has the same communication range $20 \mathrm{~m}$.

With the number of sensor nodes is 200 and 300, also under the circumstance that the anchor nodes' proportion is changed gradually, the error statics of the classic CPE localization algorithm and the improved CPE localization algorithm put 
forward by this paper is show in Figure 3 and Figure 4. Simulation result show that the improved CPE localization algorithm outperform the CPE localization algorithm significantly in estimation accuracy with different anchor nodes proportion applied. When the percentage of anchor nodes is small, the average localization error decreases as the percentage of anchor nodes increases. This is that anchor nodes can reduce the size of the unknown node's ER and thus decreases the average localization error. Our scheme performs better than the CPE scheme because we use the two-hop anchor nodes information to reduce the area of the possibility exiting area of the unknown and thus increase the accuracy of our scheme. When the number of anchor nodes becomes large, the size of ER cannot be greatly reduced. Besides, the effect of two-hop anchor nodes also become smaller and the thus the average localization to the two schemes become close to each other.

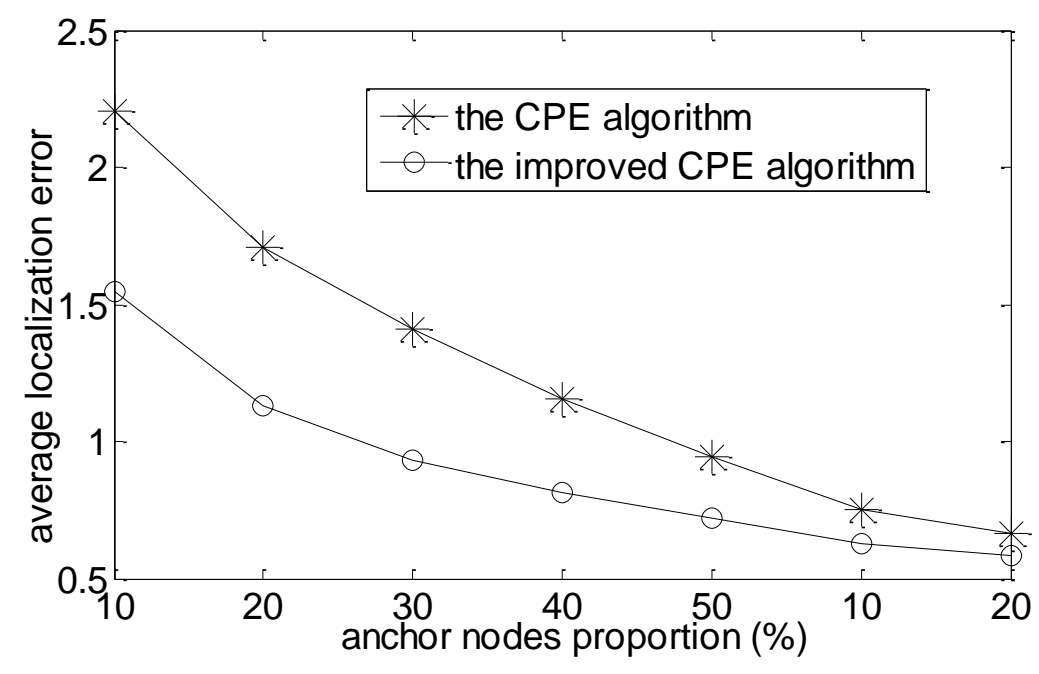

Figure 3. Anchor Nodes Proportion versus Average Localization Error (the Number of Total Nods is 200)

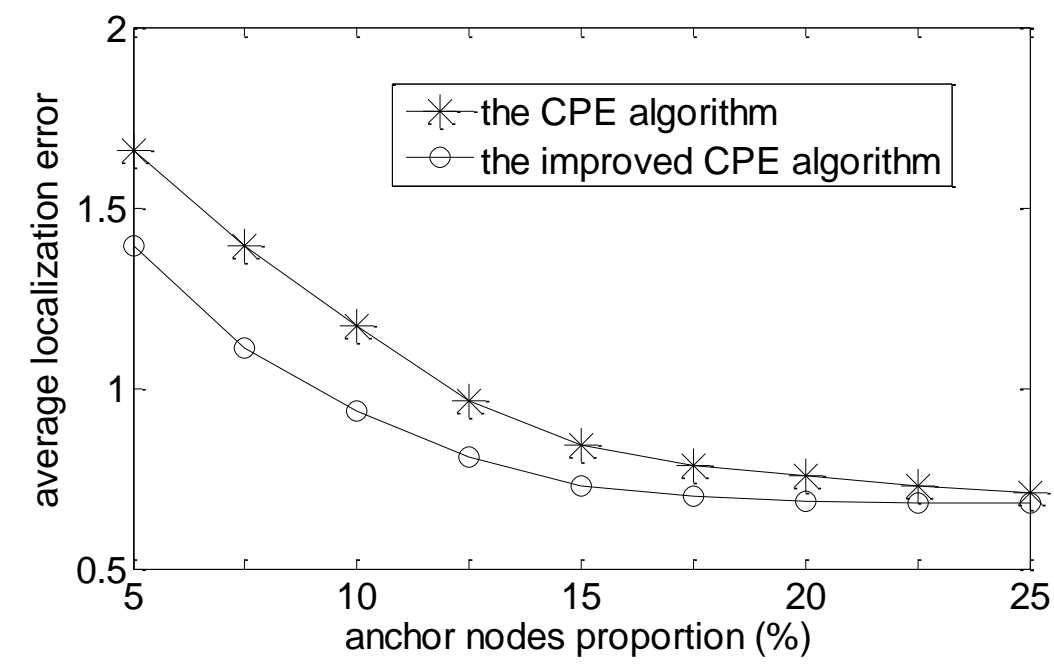

Figure 4. Anchor Nodes Proportion Versus Average Localization Error (the Number of Total Nods is 300) 


\section{Conclusions}

CPE localization algorithm is a typical and commonly used range-free localization algorithm, which has a drawback of low localization precision. In this paper, we propose a novel distributed localization algorithm using two-hop anchor nodes. In the proposed algorithm, we not only use neighboring anchors node's location to estimate the unknown nodes' position, but also we use the two-hop anchor nodes location to reduce the error of the unknown nodes' position. It has been shown via the simulation results that the improved CPE localization algorithm outperforms the classic CPE localization algorithm, and that this advantage does not increase the hardware cost of sensor nodes.

\section{References}

[1] I. F. Akyildiz, W. Su, Y. Sankarasubramaniam and E. Cayirci, "Wireless Sensor Networks: a Survey", Computer Networks, vol. 38, no.4, (2002), pp. 393-422.

[2] D. X. Ma, J. Ma and P. M. Xu, et al., "The Application Research Progress of Wireless Sensor Networks", Applied Mechanics and Materials, vol. 475, (2014), pp. 520-523.

[3] G. Han, H. Xu, T. Q. Duong et al., "Localization Algorithms of Wireless Sensor Networks: a Survey", Telecommunication Systems, vol.52, no.4, (2013), pp. 2419-2436.

[4] V. Vukadinovic and S. Mangold, "Performance of Collaborative GPS Localization in Pedestrian Ad Hoc Networks", Proceedings of the 3rd ACM international workshop on Mobile Opportunistic Networks. ACM, (2012) March, pp. 45-52.

[5] H. Wymeersch, J. Lien and M. Z. Win, "Cooperative Localization in Wireless Networks. Proceedings of the IEEE, 97.2, (2009), pp. 427-450.

[6] H. Yi and H. Aiqun, "Survey on Localization Algorithms for Wireless Sensor Networks", Telecommunications Science, vol. 26, no.7, (2010), pp. 69-75.

[7] L. Gao and Z. M. Li, "A Survey on Localization Algorithms in Wireless Sensor Networks", Applied Mechanics and Materials, vol. 530, (2014), pp. 15-18.

[8] L. Girod and D. Estrin, "Robust Range Estimation Using Acoustic and Multimodal Sensing", Proceedings of 2001 IEEE/RSJ International Conference on Intelligent Robots and Systems, vol. 3, (2001), pp. 1312-1320.

[9] A. Harter, A. Hopper and P. Steggles, et al., "The anatomy of a Context-aware Application", Wireless Networks, vol. 8, no. 2, (2002), pp. 187-197.

[10] L. Girod, V. Bychkovskiy and J. Elson, et al, "Locating Tiny Sensors in Time and Space: A Case Study", Proceedings of 2002 IEEE International Conference on Computer Design: VLSI, (2002), pp. 214-219.

[11] D. Niculescu, B. Nath, "Ad Hoc Positioning System (APS) using AOA", Proceedings of the $22^{\text {nd }}$ Annual Joint Conference of the IEEE Computer and Communications Societies, vol.3, (2003), pp. 1734-1743.

[12] N. Bulusu N, J. Heidemann and D. Estrin, "GPS-less Low Cost Outdoor Localization for Very Small Devices", IEEE Personal Communications Magazine, vol.7, no. 5, (2000), pp. 28-34

[13] L. Doherty, K. S. J. Pister, and L. E. Gaoui, "Convex Position Estimation in Wireless Sensor Networks", Proceedings of the $20^{\text {th }}$ Annual Joint Conference of the IEEE Computer and Communications Societies, vol.3, (2001) April, pp. 1655-1633.

[14] D. Niculescu D, B.Nath B, "DV Based Positioning in Ad hoc Networks", Journal of Telecommunication Systems, vol. 22, no. 1, (2003), pp. 267-280.

[15] T. He, C. D. Huang and B. Blum, et. al., "Range-free Localization Schemes for Large Scale Sensor Networks", Proceedings of the $9^{\text {th }}$ Annual International conference on Mobile Computing and Networking, (2003), pp. 81-95

[16] Y. Shang, W. Ruml and Y. Zhang, et al., "Localization from Mere Connectivity”, Proceedings of the $4^{\text {th }}$ ACM Int. Symposium on Mobile Ad Hoc Networking and Computing, Annapolis, (2003), pp. 201-212.

[17] Y. Shang and W. Ruml, "Improved MDS-based Localization. Proceedings of the $23^{\text {rd }}$ Annual Joint Conference of the IEEE Computer and Communications Societies", vol. 4, (2004), pp. 2640-2651. 
International Journal of Future Generation Communication and Networking Vol. 8, No. 1 (2015) 